\title{
Length and Roughness Perception in a Moving-Plateau Touch Display
}

\author{
Junji Watanabe, ${ }^{1}$ Yusuke Godai, ${ }^{2,3}$ and Hideyuki Ando ${ }^{4}$ \\ ${ }^{1}$ NTT Communication Science Laboratories, Nippon Telegraph and Telephone Corporation, 3-1, \\ Morinosato-Wakamiya, Atsugi, Kanagawa 243-0198, Japan \\ ${ }^{2}$ Graduate School of Electro-Communications, University of Electro-Communications, 1-5-1, \\ Chofugaoka, Chofu, Tokyo 182-8585, Japan \\ ${ }^{3}$ Machinetool Division, Mayekawa Electric. Co., Ltd., 2000, Tatsuzawa, Moriya, Ibaraki 302-0118, Japan \\ ${ }^{4}$ Graduate School of Information Science and Technology, Osaka University, 2-1, Yamadaoka, Suita, Osaka 565-0871, Japan
}

Correspondence should be addressed to Junji Watanabe, watanabe.junji@lab.ntt.co.jp

Received 13 August 2011; Revised 6 December 2011; Accepted 28 December 2011

Academic Editor: Arun Kumar Tripathi

Copyright () 2012 Junji Watanabe et al. This is an open access article distributed under the Creative Commons Attribution License, which permits unrestricted use, distribution, and reproduction in any medium, provided the original work is properly cited.

\begin{abstract}
We have proposed a tactile geometry display technique based on active finger movement. The technique uses a perceptual feature that, during finger movement, the length of a touched object is perceived to increase when the object is moved in the same direction as the finger movement or to decrease when it is moved in the opposite direction. With this display technique, a wide range of tactile shapes can be presented with realistic rigid edges and continuous surfaces. In this work, to further develop our technique, we performed psychophysical experiments to study perceptions of length and roughness under this presentation technique. The results indicated that the elongation (shrinkage) of the object can be observed regardless of the roughness of the touched object and that the perceived roughness of the object slightly changes but the changes are much smaller than those theoretically expected.
\end{abstract}

\section{Introduction}

1.1. Moving-Plateau Touch Display. When we touch an object in a space, we actively move our fingers across its surface. Since the cutaneous signals sequentially presented to the fingertips are integrated with proprioceptive signals of the finger and arm, we can perceive the shape and texture of the object, even if it is larger than the fingertips. The interplay of the cutaneous and proprioceptive sensations is essential for developing touch displays, and we have proposed a novel touch display technique for presenting tactile geometry based on the perceptual features of the two sensory processes $[1-3]$. Touched objects do not usually move during exploratory finger movements, but if the object moves during the finger movement, an illusory shape of the object can be perceived. As shown in Figure 1(a), when the object moves in the same direction as the finger, the length of the object is perceived to increase. Conversely, when the object moves in the opposite direction as in Figure 1(b), its length is perceived to decrease. This is because the human tactile system judges the length based on the initial and final positions of the moving edges, even if the information about the object's movement exists on the fingertip. In this touch display technique, called the Moving-Plateau Touch Display (MPTD), the extent of the change in the perceived length can be controlled by manipulating the velocity of the object during the finger movement. In this paper, we verify the generality of our display technique from the results of psychophysical experiments on the perceptions of length and roughness under the MPTD presentation.

1.2. Related Touch Displays and Characteristics of MPTD. To clarify the characteristics of the MPTD, here we briefly introduce previous touch displays for presenting tactile surfaces and shapes. In a pin array display, pins are arranged as a matrix on a two-dimensional plane and the pins independently rise and fall [4-7]. Although a highdensity pin matrix display has been developed [8], pinbased displays cannot present a smooth continuous surface, 


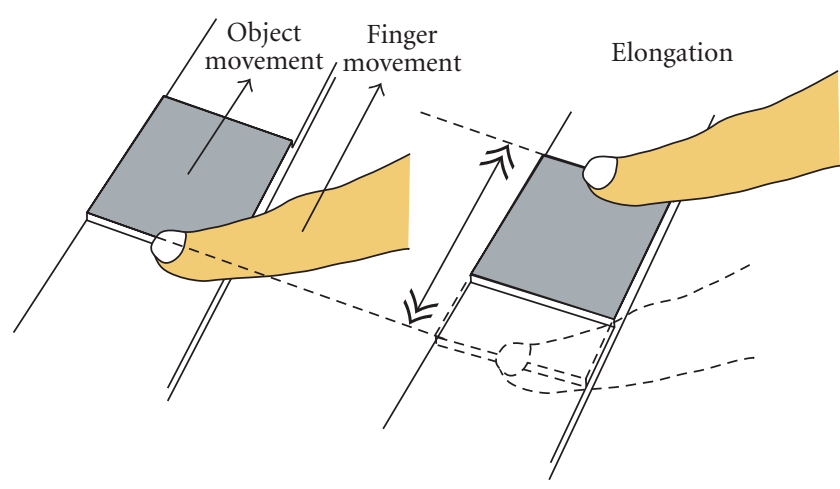

(a)

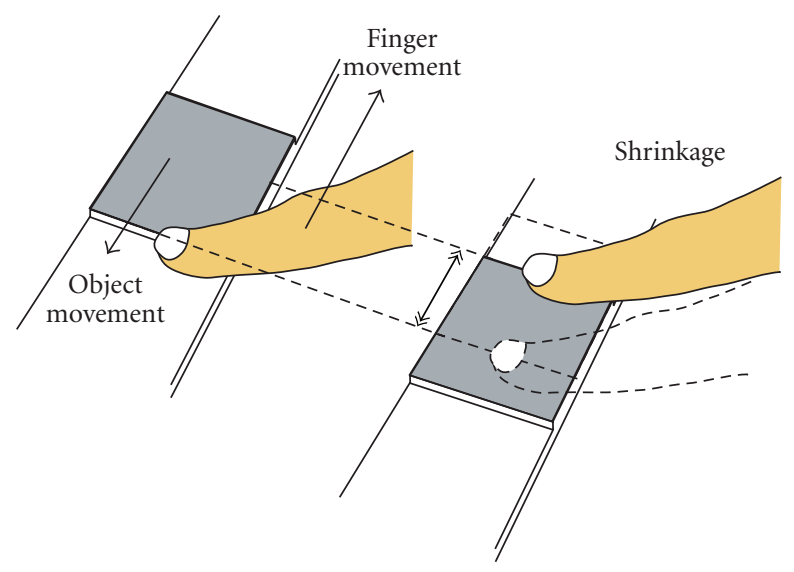

(b)

FIGURE 1: The principle of MPTD. (a) When the object moves in the same direction as the finger, the length is perceived to increase. (b) When it moves in the opposite direction, the length is perceived to decrease.

which is essential for achieving natural tactile information presentation. In probe-based displays, such as Phantom [9] and Spider [10], the contact point is a pen or sphere connected to force generator. These displays can present smooth proprioceptive sensation, but can not present a cutaneous one. In an encounter-type display [11, 12], the display stays at the location of a virtual object and waits for the user to encounter it. With such devices, there is no need to prepare the whole surface in the environment, but contact situations are limited or mechanical probes have to be used to present the object's surface. As an extension of the encountered-type displays, a plate [13], rotating drum [14], or moving centroid [15] has been used to present local orientation or slips at the location of the contact point with the fingertip, which can produce both proprioceptive and cutaneous sensations and significantly enhance perception of shape and surface.

The MPTD is another variation of the encounter-type display. The MPTD was implemented by using a linear slider, an object attached to the slider, and a laser distance sensor for measuring finger movements. When the user touches an object on the slider, the MPTD can change the position of the object according to the finger movements measured with the distance sensor and thereby present tactile geometric information. The MPTD can present not only realistically rigid edges but also continuous surfaces, which are difficult for prior tactile displays. In addition, this technique can easily be extended to a two-dimensional shape by moving the object along two orthogonal axes (see Figure 2 and video on the website [3]).

In the MPTD presentation, it has been demonstrated that perceived length is changed by moving the object on the slider during finger movements [1-3]. However, the relationship between the effect of the MPTD and roughness of the object remains unclear. In this work, we tested the gene-rality and effectiveness of the MPTD by conducting psychophysical experiments to determine (i) whether the perceived length changes with the roughness of the object

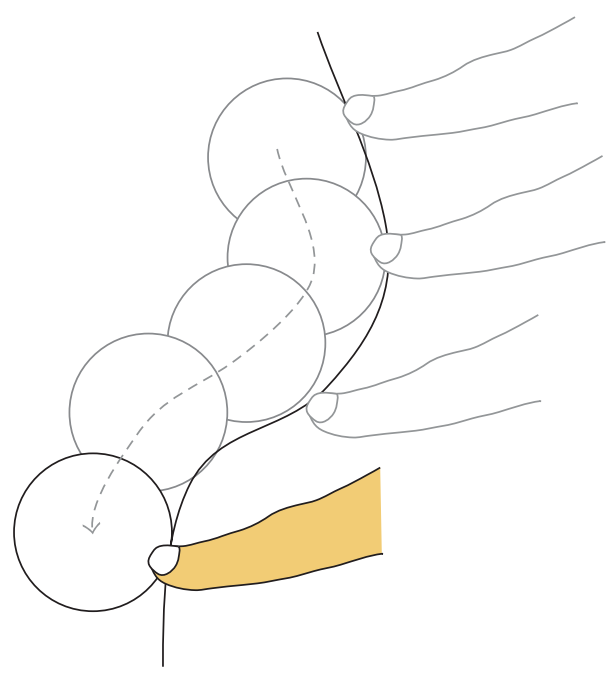

FIGURE 2: Principle for presenting smooth two-dimensional shapes in the MPTD presentation. The touched object is moved on the two orthogonal axes according to the finger movements.

and (ii) whether perceived roughness changes when the length is modulated by the MPTD.

\section{Materials and Methods}

2.1. Participants. Six people (one male author, three naïve males, and two naïve females, who were all right handed and in their twenties) participated in all experiments. The participants, excluding the author, were volunteers unaware of the purpose of the experiments. They had no known abnormalities of their tactile sensory systems. Informed consent was obtained from the naïve participants before the experiment started. Recruitment of the participants and experimental procedures were conducted in accordance with the Declaration of Helsinki. 
2.2. Apparatus. The participant traced an object placed on a linear slider (KBA-10E-ST-M20N-20A, CKD Inc.), whose motor had been replaced with a high-response motor (MSMD041S1A, Panasonic Inc.). The finger position of the participant was measured with a laser range finder (LK-500, KEYENCE Corp.), and its information was sent to a PC. The data were collected with the PC using an AD board (PCI-3523A, Interface Corp.), and the finger velocity was calculated. The liner slider was driven by a DA board (PCI3523A, Interface Corp.) of the PC at a 1-kHz cycle.

2.3. Stimuli. Past tactile experiments used a variety of roughness samples, such as abrasive surfaces (e.g., $[16,17])$, onedimensional gratings (e.g., $[18,19]$ ), and two-dimensional dot patterns (e.g., $[20,21])$. However, the randomness of the roughness parameters makes it difficult to quantitatively evaluate the experimental results. Our solution is to use original plastic samples. As illustrated in Figure 3(a), we wrapped nylon string around a flawless round bar and made a casting mold of the string-wrapped bar with silicon. Figure 3(b) is an example of the casting molds. Using the casting mold, we made a roughness sample of a onedimensional grating with casting resin. A photograph of the roughness sample and its cross-section are shown in Figure 4(a) and 4(b), respectively. In our experiments, the roughness is expressed by the diameter of the nylon string (e.g., 1.0-mm diameter (mm-d)). A smooth surface (0 mmd) was made from the mold of a bare round bar. The length of roughness surface was adjusted by covering flanking areas with masking tapes.

2.4. Length and Roughness Configuration. The velocity of the object on the linear slider $V_{o}$, the expected length $L_{e}$, the expected roughness $R_{e}$ expressed in mm-d, and their ratio to the original ones $X$ are calculated by

$$
\begin{gathered}
V_{o}=\alpha V_{f}(\alpha<1), \\
L_{e}=\frac{V_{f}}{V_{f}-V_{o}} \times L, \\
R_{e}=\frac{V_{f}}{V_{f}-V_{o}} \times R, \\
X=\frac{1}{(1-\alpha)},
\end{gathered}
$$

where $V_{f}$ is the velocity of the finger movement, $\alpha$ is the ratio of object's velocity to finger's velocity, and $L$ and $R$ are the original length and roughness of the touched object, respectively. If the object does not move, that is, $\alpha=0$, the veridical length and roughness can be perceived $(X=1.0)$. If the object moves in the same direction as the finger at half the speed of the finger movement, that is, $\alpha=0.5$, since the number of bumps on the object is constant, twice the original length and roughness can be perceived $(X=2.0)$. If the object moves at the same speed in the opposite direction, that is, $\alpha=-1.0$, half of the original length and roughness can be perceived $(X=0.5)$.

\section{Perceived Lengths with Varied Roughness}

3.1. Procedure. A participant sat in front of the linear slider. A standard length sample (length of $40 \mathrm{~mm}$, and roughness of $0.00,0.14,0.40$, or $1.00 \mathrm{~mm}-\mathrm{d}$ ) was put on the forward side of the linear slider. A test length sample (32 samples, length of 16 - to 90 -mm-length at 2 -mm interval, and the same roughness as the standard sample) was put on the interior side of the linear slider. First, the participant traced the test length sample from the left starting position to the right end position once as in Figure 5(a). At this time, the linear slider did not move. Then, the participant traced the standard length sample from the left starting position to the right end position once as in Figure 5(b). At this time, the linear slider moved at a relative velocity rate $\alpha$ defined in (1). Then, the participant verbally indicated which of length samples he/she perceived to be longer by an alternative forced choice task (2AFC). Next, another test length sample was set up based on the answer. We selected the test length sample using the simple up-and-down method. For example, a test sample reported as longer was replaced with one whose length was $2 \mathrm{~mm}$ shorter. Then, the shorter test sample was presented until the participant reported that it was shorter. This procedure was repeated until the answer changed three times. We regarded the length of the last test sample as the point of subjective equality (PSE) of perceived length. We selected five velocity ratios $(\alpha=-0.67,-0.25$, $0.00,0.20$, and 0.50 ) of the linear slider at random. The expected values of perceived length calculated from (2), their differences from the original lengths, and their ratios to the original ones calculated from (4) for the five velocity ratios are shown in Table 1. The participant tried the simple upand-down method four times at each velocity ratio (total of 20 times). In addition, we conducted experiments with four roughness conditions $(0.00,0.14,0.40$, and $1.00 \mathrm{~mm}$ d).

To eliminate any distraction caused by sound noise (motor noise and tracing sounds), the participants wore both earplugs (ear putty, KOKEN) and ear muffs (Leightning L3, Bilsom). Participants' eyes were always closed. The participants practiced before the experiment to attain a constant finger movement velocity of $30 \mathrm{~mm} / \mathrm{sec}$ and constant finger pressure of $50 \mathrm{~g}$. In order to keep the regular speed of the finger movement, they moved their finger according to metronome (120 beats per minute). When the participants were aware of a failure in the finger movement, they were asked to report it, and the trial was performed again.

3.2. Results. The experimental results for the four roughness conditions $(0.00,0.14,0.40$, and $1.00 \mathrm{~mm}-\mathrm{d})$ are shown in Figure 6 . The horizontal axis represents the velocity ratio of the liner slider $\alpha$ and the vertical axis represents the differences of the PSE of perceived length from the original length. Averages of perceived lengths across all participants and their standard errors are shown. Dotted lines show theoretical values calculated from (2).

The results for the four roughness conditions showed similar tendencies. In order to evaluate the statistical difference 


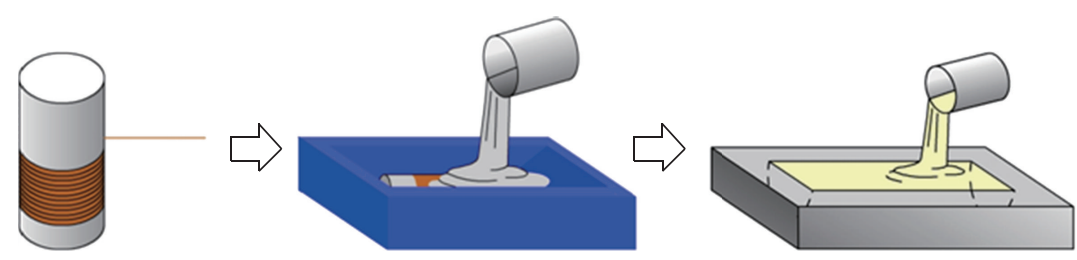

(a)
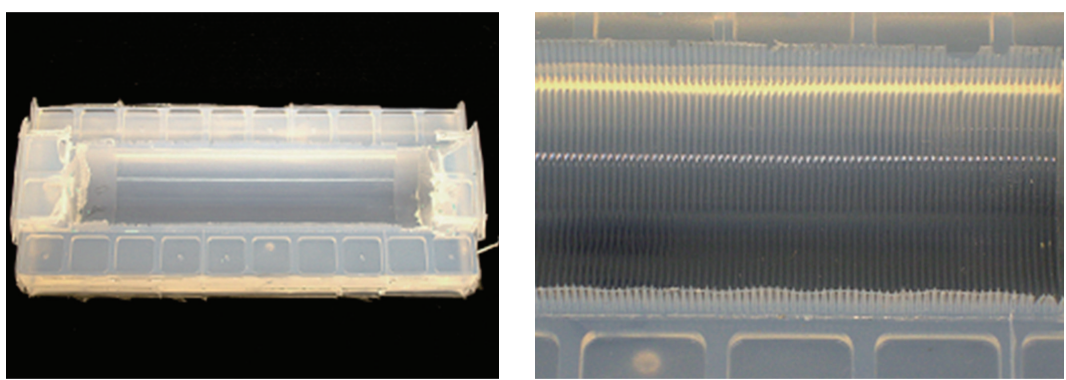

(b)

Figure 3: (a) Illustrations showing how a roughness sample is made. (b) Photograph of casting mold.

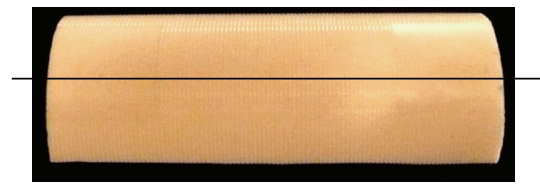

(a)

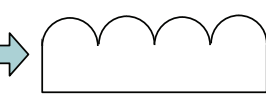

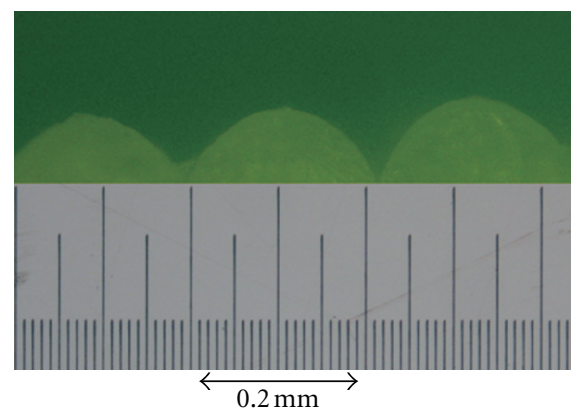

(b)

Figure 4: (a) Photograph of roughness sample. (b) Photograph of cross-sectional surface of the roughness sample (0.20 mm-d). This sample was cut with a band saw.

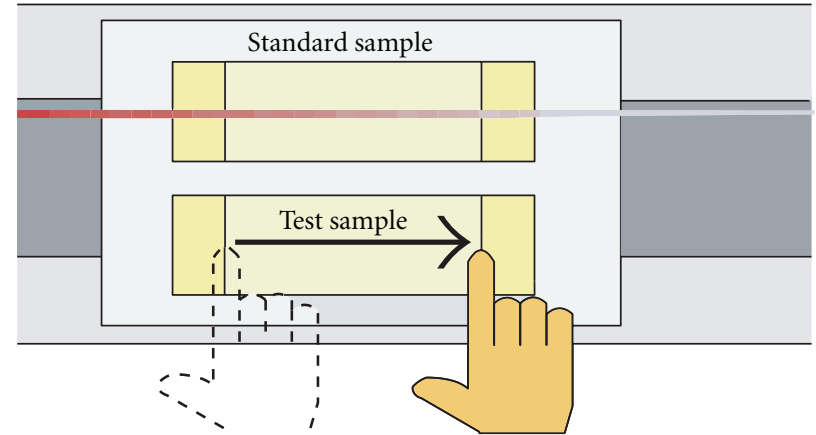

Finger movement

(a)

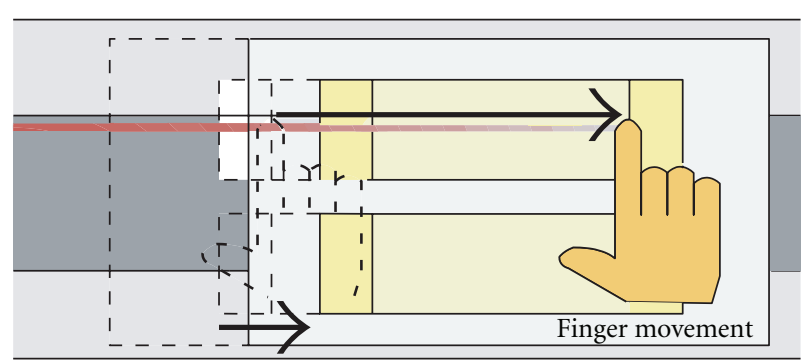

Object movement

Figure 5: Experimental procedures. (a) The participant traced the test length sample while the linear slider was static. (b) The participant traced the standard length sample while the linear slider was moved at a relative velocity rate $\alpha$. 
TABLE 1: Theoretically expected length $L_{e}(\mathrm{~mm})$, difference of $L_{e}$ from original length $(40 \mathrm{~mm})$, and its ratio $X$ to original according to velocity ratio $\alpha$.

\begin{tabular}{lccccc}
\hline Velocity ratio $\alpha$ & -0.67 & -0.25 & 0.00 & 0.20 & 0.50 \\
\hline Expected length $L_{e}(\mathrm{~mm})$ & 24 & 32 & 40 & 50 & 80 \\
\hline $\begin{array}{l}\text { Difference of } L_{e} \text { from } \\
\text { original length }(\mathrm{mm})\end{array}$ & -16 & -8 & 0 & +10 & +40 \\
\hline Ratio to original $X$ & 0.60 & 0.80 & 1.00 & 1.25 & 2.00 \\
\hline
\end{tabular}

in the PSEs, we first performed a two-way repeated measures ANOVA with the roughness condition and velocity ratio as factors, and the PSE of perceived length as the dependent variable. The statistical results showed a nonsignificant difference between roughness conditions $(F(3,15)=1.13$, $P=0.37)$ and a significant difference between velocity ratios $(F(4,20)=155.26, P<.01)$. The interaction between them was not significant $(F(12,60)=1.06, P=0.41)$. The post hoc multiple comparisons (Ryan's method, significant level $=0.05$ ) showed significant differences between all combinations of velocity ratios (see Remark 1 for the statistical analyses excluding the author's data). These results suggest that the perceived lengths change according to the velocity ratios and that the roughness of the traced object does not influence the perceived length under the MPTD presentation.

Since a significant difference was not observed in the roughness conditions, we use all data of the four roughness conditions without distinction for the following analyses. When the velocity ratio was zero (the standard length sample did not move), almost veridical length was perceived (average $39.3 \mathrm{~mm}$ ), and the value was not significantly different from the theoretical one $(40 \mathrm{~mm})(t(23)=1.34, P=0.19)$, suggesting that the participants could judge the length correctly. In order to avoid artifacts due to the presentation order of standard and test samples, we subtracted the value of velocity ratio $\alpha=0.00$ from those of the rest and then made comparisons with the theoretical values. When the object was moved during a finger movement, the magnitudes of elongation or shrinkage fell short of the theoretical ones: theoretical value of $79.9 \%$ for $\alpha=-0.67,74.1 \%$ for $\alpha=$ $-0.25,88.7 \%$ for $\alpha=0.20$, and $75.5 \%$ for $\alpha=0.50$. These results indicate that the changes in perceived lengths are associated with the velocity ratios and that the magnitudes of elongation or shrinkage of them are slightly smaller than the theoretical ones.

Remark 1. The statistical results remained the same even when the author's data were excluded. A two-way repeated measures ANOVA showed a nonsignificant difference between roughness conditions $(F(3,12)=0.76, P=0.54)$ and a significant difference between velocity ratios $(F$ ( 4 , 16) $=113.44, P<.01)$. The interaction between them was not significant $(F(12,48)=0.97, P=0.50)$. The post hoc multiple comparisons (Ryan's method, significant level $=0.05$ ) showed significant differences between all combinations of velocity ratios.

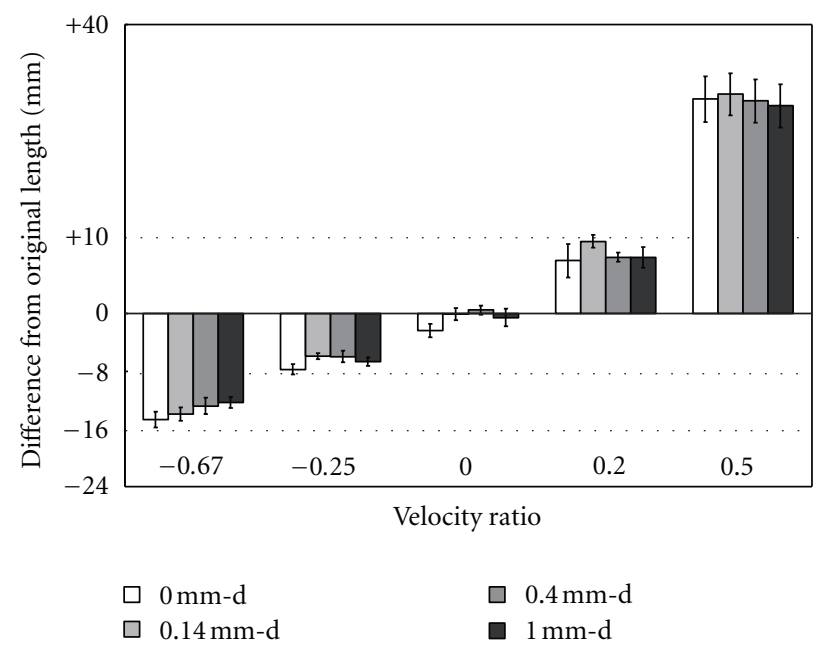

Figure 6: Differences in the PSEs of perceived length from the original length in $0.00,0.14,0.40$, and $1.00-\mathrm{mm}$-d roughness conditions. Dotted lines show theoretical values calculated from (2).

\section{Perceived Roughness under MPTD Presentation}

4.1. Procedure. In this experiment, we investigated the roughness perception under the MPTD presentation. The apparatuses were the same as in the previous experiment. The standard roughness sample (length of $40 \mathrm{~mm}$, and roughness of 0.4 or $0.6 \mathrm{~mm}-\mathrm{d}$ ) was put on the forward side of the linear slider. The test roughness sample (15 samples, length of $40 \mathrm{~mm}$, and roughness of 0.20 - to $0.90-\mathrm{mm}-\mathrm{d}$ at a $0.05-\mathrm{mm}$-d interval) was put on the interior side of the linear slider. First, the participant traced the test roughness sample once. At this time, the linear slider was stationary. Then, the participant traced the standard roughness sample once. At this time, the linear slider moved at a relative velocity ratio $\alpha$. The participant verbally indicated which sample he/she perceived to be rougher by 2AFC. Next, another test sample was set on the linear slider using the same up-and-down method as in the previous experiment. We regarded the roughness of the last test sample as the PSE of perceived roughness. We used the five velocity ratios $(\alpha=-0.67$, $-0.25,0.00,0.20$, and 0.50 ) of the linear slider at random. The participant tried the up-and-down method four times at each velocity ratio (total of 20 times). In addition, we conducted experiments with two roughness conditions $(0.40$ and $0.60 \mathrm{~mm}-\mathrm{d})$. The theoretically expected roughness for 0.40 and $0.60 \mathrm{~mm}-\mathrm{d}$ calculated from (3), and their ratios to the original ones calculated from (4) for the five velocity ratios are shown in Table 2.

4.2. Results. Figure 7 shows the averages of the PSEs of perceived roughness across all participants and their standard errors for the two roughness conditions $(0.4$ and $0.6 \mathrm{~mm}-\mathrm{d})$. The horizontal axis represents the velocity ratio of the linear slider $\alpha$, and the vertical axis represents the PSE of perceived roughness. Perceived roughness (solid line) and theoretically expected values (dotted line) are plotted. 
TABLE 2: Theoretically expected roughness $R_{e}(\mathrm{~mm}-\mathrm{d})$ and its ratio $X$ to the original roughness $(0.40$ and $0.60 \mathrm{~mm}-\mathrm{d})$ according to velocity ratio $\alpha$

\begin{tabular}{lccccc}
\hline Velocity ratio $\alpha$ & -0.67 & -0.25 & 0.00 & 0.20 & 0.50 \\
\hline $\begin{array}{l}\text { Expected roughness } R_{e} \\
(\mathrm{~mm}-\mathrm{d}) \text { for } 0.40 \mathrm{~mm}-\mathrm{d}\end{array}$ & 0.24 & 0.32 & 0.40 & 0.50 & 0.80 \\
\hline $\begin{array}{l}\text { Expected roughness } R_{e} \\
(\mathrm{~mm}-\mathrm{d}) \text { for } 0.60 \mathrm{~mm}-\mathrm{d}\end{array}$ & 0.36 & 0.48 & 0.60 & 0.75 & 1.20 \\
\hline Ratio to original $X$ & 0.60 & 0.80 & 1.00 & 1.25 & 2.00 \\
\hline
\end{tabular}

The same trend was observed in the two roughness conditions that when the velocity ratio increased, the PSEs of perceived roughness were slightly increased. In order to evaluate the statistical difference in the PSEs, we first performed a two-way repeated measures ANOVA with the roughness condition and velocity ratio as factors, and the PSE of perceived roughness as the dependent variable. The statistical results showed significant differences between roughness conditions $(F(1,5)=58.93, P<.01)$ and between velocity ratios $(F(4,20)=7.02, P<.01)$. The interaction between them was not significant $(F(4,20)=$ $0.58, P=0.68$ ). The post hoc multiple comparisons (Ryan's method, significant level $=0.05$ ) showed that there were significant differences between following combinations of velocity ratios: $[\alpha=-0.67$ and 0.20$],[\alpha=-0.67$ and 0.50$]$, and $[\alpha=-0.25$ and 0.50$]$ (see Remark 2 for the statistical analyses excluding the author's data).

To evaluate the magnitude of the change in roughness with the changes in velocity ratios, we subtracted the value of velocity ratio $\alpha=0.00$ from those of the rest, and then compared the differences with the theoretical values. When the object was moved during a finger movement, the changes in perceived roughness were smaller than the theoretical ones in all velocity ratios in the two roughness conditions. The proportions to the theoretical ones were $41.5 \%$ for $\alpha=$ $-0.67,38.2 \%$ for $\alpha=-0.25,15.3 \%$ for $\alpha=0.20$, and $17.0 \%$ for $\alpha=0.50$ in $0.4 \mathrm{~mm}-\mathrm{d}$ roughness condition, and $26.8 \%$ for $\alpha=-0.67,26.0 \%$ for $\alpha=-0.25,42.1 \%$ for $\alpha=0.20$, and $11.2 \%$ for $\alpha=0.50$ in $0.6 \mathrm{~mm}$-d roughness condition. These results suggest that, although the perceived roughness could be changed according to the changes in velocity ratios, the changes were much smaller than those expected by theory (less than half in all velocity ratios).

Remark 2. When the author's data were excluded, we found less effect of the velocity ratios on the perceived roughness than when the data were included. A two-way repeated measures ANOVA showed significant differences between roughness conditions $(F(1,4)=40.44, P<.01)$ and between velocity ratios $(F(4,16)=4.57, P<.05)$. The interaction between them was not significant $(F(4,16)=0.59, P=$ 0.67). The post hoc multiple comparisons (Ryan's method, significant level $=0.05$ ) showed that there was a significant difference only between the combination of velocity ratios: $[\alpha=-0.67$ and 0.50$]$.

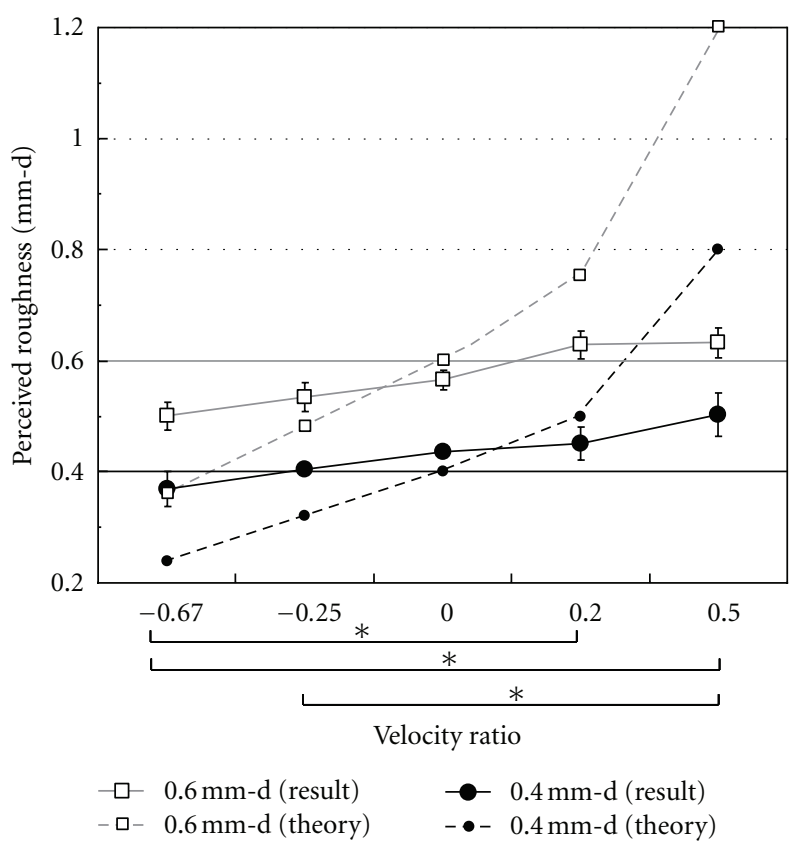

FIGURE 7: PSEs of perceived roughness in 0.40 and $0.60-\mathrm{mm}-\mathrm{d}$ roughness conditions. The theoretical values calculated from (3) are plotted with dotted lines. The significant differences between velocity ratios are also shown $\left({ }^{*} P<.05\right)$.

\section{Discussion}

5.1. Principle Findings. In the current paper, two psychophysical experiments were performed under the MPTD presentation. The first experiment examined length perception with varied roughness of touch objects, and the results demonstrated that the perceived length could be changed systematically according to the velocity ratios of object's movement and that this trend was observed regardless of the roughness of the touched object. This means that the MPTD can effectively work regardless of presented texture. The second experiment examined roughness perception when the perceived length was changed, and the result indicated that, although the perceived roughness was modulated by the movements of the touched object, the changes in perceived roughness were much smaller than theoretically expected values. This suggests that the texture of touched object might be modulated but not dramatically changed when the MPTD is used for presenting geometric information.

5.2. Perceptions of Length and Roughness under MPTD. Since our experiments were performed without visual guidance, only the proprioceptive signal of the finger movement and the cutaneous signal on the fingertip were available for perceiving the information about touched object. In the first experiment, the systematic increase/decrease of perceived length was observed regardless of a variety of relative movements between the skin on the fingertip and object, which were caused by the movements of the finger and touched object. In addition, the perceived length of the touched object was comparable to the travel distance of 
the finger even when the physical roughness of the object was changed. These results suggest that the proprioceptive signals of finger locations at the initiation and end of finger movement are dominant determinants for judging the length of an object in our experimental condition, where the direction and velocity of the finger movement were constant. Considering that the perceived length could be changed depending on the direction [22] and velocity [23,24] of the finger movement, their roles under the MPTD presentation would be an issue awaiting further investigation.

The results of second experiment indicated that the difference in the relative velocity between the skin and object had little influence on the perceived roughness. This trend agrees with the previous findings with active exploration of one-dimensional gratings [18], two-dimensional raiseddot patterns of truncated cones [25] and textured material surfaces [26], and with passive touch of two-dimensional raised-dot patterns [27]. In our experiment, the participant could not perceive the roughness before or after the finger movement, since the flanking areas of the roughness surface were covered with masking tapes, suggesting that the roughness was not judged when the finger was static. Meanwhile how the participant judged the roughness during the finger movement was not clear. The participant could judge the roughness during any period of the finger movement. It is also known that the sensitivity could be somehow degraded during a finger movement [28]. How the surface roughness is perceived under the MPTD remains for a future study.

The computational mechanisms of the interplay between proprioceptive and cutaneous sensory signals are currently attracting attention in scientific studies of touch (e.g., studies on temporal processing $[29,30]$, motion processing [31, 32], thermal processing [33], and time course of the interplay [34]), and the MPTD might be an interesting display system for psychophysically investigating mechanisms underlying these sensory processing.

5.3. Future Prospect of MPTD. As mentioned in the introduction, the MPTD can easily be extended to a two-dimensional shape display by moving the object along two orthogonal axes. In addition to this tactile function, a visual image presentation function can be integrated (see also video on the website [3]). For example, as shown in Figure 8, when a piece of transparent material is placed on a transparent screen and the image is projected from the back of the screen, the edge of the tactile object on the screen can align with the edge of the visual image. When the user moves his/her finger on the static visual image, the transparent tactile material is moved synchronously according to the shape of the visual image. Then, the visual and tactile edges of the object can overlap.

\section{Conclusion}

To clarify the effectiveness of the MPTD with objects of different roughness, we conducted two psychophysical experiments. In the first experiment, we examined the relationship between physical roughness of the object and its perceived length and found that the roughness of the touched



FIGURE 8: Principle for achieving a visual-tactile function with the MPTD.

object does not influence perceived length. In the second experiment, we examined perceived roughness when the length is presented by the MPTD and found that the MPTD influences the perception of the object's roughness, but the changes in perceived roughness are much smaller than those theoretically expected. From these experiments, we can conclude that the MPTD can present geometric information for a wide range of object, and that, when the MPTD is used, the information can be presented without a drastic distortion of its roughness.

\section{References}

[1] H. Ando, T. Amemiya, T. Maeda, J. Watanabe, and M. Nakatani, "Embossed touch display: illusory elongation and shrinking of tactile objects," in Proceedings of the International Conference on Computer Graphics and Interactive Techniques, Emerging Technologies (SIGGRAPH '06), Boston, Mass, USA, July 2006.

[2] H. Ando, J. Watanabe, T. Amemiya, and T. Maeda, "The two dimension outline shape display device using active touch," in Proceedings of the 16th International Conference on Artificial Reality and Telexistence (ICAT), Special Workshop, p. 3A1-1, Hangzhou, China, December 2006.

[3] http://www.junji.org/mptd/.

[4] H. Iwata, H. Yano, F. Nakaizumi, and R. Kawamura, "Project FEELEX: adding haptic surface to graphics," in Proceedings of the Computer Graphics Annual Conference (SIGGRAPH '01), pp. 469-475, Los Angels, Calif, USA, August 2001.

[5] P. S. Wellman, W. J. Peine, G. Favalora, and R. D. Howe, "Mechanical design and control of a high-bandwidth shape 
memory alloy tactile display," in Proceedings of the 5th International Symposium on Experimental Robotics V, vol. 232, pp. 56-66, Barcelona, Spain, June 1997.

[6] J. Lee, I. Ahn, and J. Park, "Design and implementation of tactile feedback device using electromagnetic type," in Proceedings of the Intelligent Robots and Systems (IROS '99), vol. 3, pp. 1549-1554, Kyongju, Korea, October 1999.

[7] C. R. Wagner, R. D. Howe, and S. J. Lederman, "A tactile shape display using RC servomotors," in Proceedings of the 10th Symposium on Haptic Interfaces for Virtual Environment and Teleoperator Systems, p. 354, Orlando, Fla, USA, March 2002.

[8] M. Shimojo, M. Shinohara, and Y. Fukui, "Human shape recognition performance and pin-matrix density in a 3 dimensional tactile display," in Proceedings of the 5th IEEE International Workshop on Robot and Human Communication, pp. 513-518, Tsukuba, Japan, November 1996.

[9] J. K. Salisbury and M. A. Srinivasan, "Phantom-based haptic interaction with virtual objects," IEEE Computer Graphics and Applications, vol. 17, no. 5, pp. 6-10, 1997.

[10] M. Ishii, M. Nakata, and M. Sato, "Networked SPIDAR: a networked virtual environment with visual, auditory, and haptic interactions," Presence, vol. 3, no. 4, pp. 351-359, 1994.

[11] S. Tachi, T. Maeda, R. Hirata, and H. Hoshino, "A construction method of virtual haptic space," in Proceedings of the 4th International Conference on Artificial Reality and Tele-existence (ICAT '94), pp. 131-138, Tokyo, Japan, July1994.

[12] Y. Yokokohji, N. Muramori, Y. Sato, and T. Yoshikawa, "Designing an encountered-type haptic display for multiple fingertip contacts based on the observation of human grasping behavior," in Proceedings of the 12th International Symposium on Haptic Interfaces for Virtual Environment and Teleoperator Systems (HAPTICS '04), pp. 66-73, Chicago, Ill, USA, March 2004.

[13] A. Frisoli, M. Solazzi, F. Salsedo, and M. Bergamasco, "A fingertip haptic display for improving curvature discrimination," Presence, vol. 17, no. 6, pp. 550-561, 2008.

[14] M. A. Salada, J. E. Colgate, M. V. Lee, and P. M. Vishton, "Fingertip haptics: a novel direction in haptic display," in Proceedings of the 8th Mechatronics Forum International Conference, pp. 1211-1220, Enschede, The Netherlands, June 2002.

[15] W. R. Provancher, M. R. Cutkosky, K. J. Kuchenbecker, and G. Niemeyer, "Contact location display for haptic perception of curvature and object motion," International Journal of Robotics Research, vol. 24, no. 9, pp. 691-702, 2005.

[16] M. Hollins and S. R. Risner, "Evidence for the duplex theory of tactile texture perception," Perception and Psychophysics, vol. 62, no. 4, pp. 695-705, 2000.

[17] T. Miyaoka, T. Mano, and M. Ohka, "Mechanisms of finesurface-texture discrimination in human tactile sensation," Journal of the Acoustical Society of America, vol. 105, no. 4, pp. 2485-2492, 1999.

[18] S. J. Lederman, "Tactual roughness perception: spatial and temporal determinants," Canadian Journal of Psychology, vol. 37, no. 4, pp. 498-511, 1983.

[19] M. Hollins, S. J. Bensmaïa, and S. Washburn, "Vibrotactile adaptation impairs discrimination of fine, but not coarse, textures," Somatosensory and Motor Research, vol. 18, no. 4, pp. 253-262, 2001.

[20] C. E. Connor and K. O. Johnson, "Neural coding of tactile texture: comparison of spatial and temporal mechanisms for roughness perception," Journal of Neuroscience, vol. 12, no. 9, pp. 3414-3426, 1992.
[21] M. Kahrimanovic, W. M. Bergmann Tiest, and A. M. L. Kappers, "Context effects in haptic perception of roughness," Experimental Brain Research, vol. 194, no. 2, pp. 287-297, 2009.

[22] M. A. Heller and T. D. Joyner, "Mechanisms in the haptic horizontal-vertical illusion: evidence from sighted and blind subjects," Perception and Psychophysics, vol. 53, no. 4, pp. 422428, 1993.

[23] M. Hollins and A. K. Goble, "Perception of the length of voluntary movements," Somatosensory Research, vol. 5, no. 4, pp. 335-348, 1988.

[24] B. L. Whitsel, O. Franzen, D. A. Dreyer et al., "Dependence of subjective traverse length on velocity of moving tactile stimuli," Somatosensory Research, vol. 3, no. 3, pp. 185-196, 1986.

[25] A. M. Smith, C. E. Chapman, M. Deslandes, J. S. Langlais, and M. P. Thibodeau, "Role of friction and tangential force variation in the subjective scaling of tactile roughness," Experimental Brain Research, vol. 144, no. 2, pp. 211-223, 2002.

[26] T. Yoshioka, J. C. Craig, G. C. Beck, and S. S. Hsiao, "Perceptual constancy of texture roughness in the tactile system," Journal of Neuroscience, vol. 31, no. 48, pp. 1760317611,2011

[27] E. M. Meftah, L. Belingard, and C. E. Chapman, "Relative effects of the spatial and temporal characteristics of scanned surfaces on human perception of tactile roughness using passive touch," Experimental Brain Research, vol. 132, no. 3, pp. 351-361, 2000.

[28] M. P. Vitello, M. O. Ernst, and M. Fritschi, "An instance of tactile suppression: active exploration impairs tactile sensitivity for the direction of lateral movement," in Proceedings of the The EuroHaptics Conference, pp. 351-355, Paris, France, July 2006.

[29] S. Yamamoto and S. Kitazawa, "Reversal of subjective temporal order due to arm crossing," Nature Neuroscience, vol. 4, no. 7, pp. 759-765, 2001.

[30] S. Kuroki, J. Watanabe, N. Kawakami, S. Tachi, and S. Nishida, "Somatotopic dominance in tactile temporal processing," Experimental Brain Research, vol. 203, no. 1, pp. 51-62, 2010.

[31] J. C. Craig, "The effect of hand position and pattern motion on temporal order judgments," Perception and Psychophysics, vol. 65 , no. 5, pp. 779-788, 2003.

[32] S. Kuroki, J. Watanabe, K. Mabuchi, S. Tachi, and S. Nishida, "Directional remapping in tactile inter-finger apparent motion: a motion aftereffect study," Experimental Brain Research, vol. 216, no. 2, pp. 311-320, 2011.

[33] H. N. Ho, J. Watanabe, H. Ando, and M. Kashino, "Somatotopic or spatiotopic? Frame of reference for localizing thermal sensations under thermo-tactile interactions," Attention, Perception, and Psychophysics, vol. 72, no. 6, pp. 1666-1675, 2010.

[34] E. Azanon and S. Soto-Faraco, "Changing Reference Frames during the Encoding of Tactile Events," Current Biology, vol. 18, no. 14, pp. 1044-1049, 2008. 

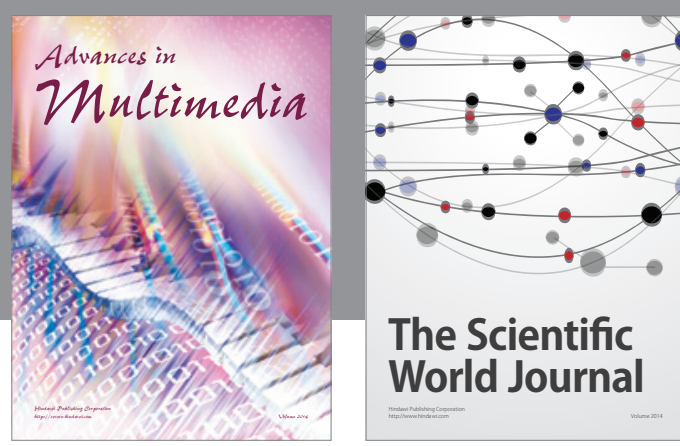

The Scientific World Journal
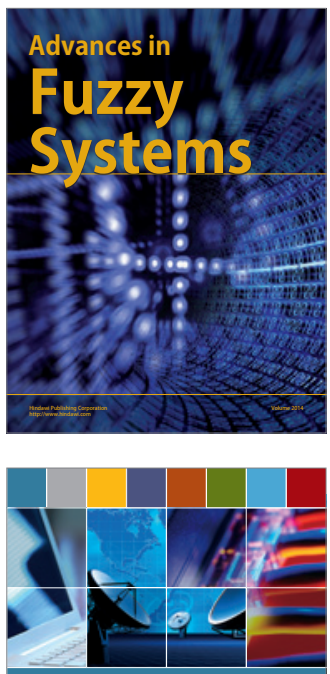

Computer Networks and Communications
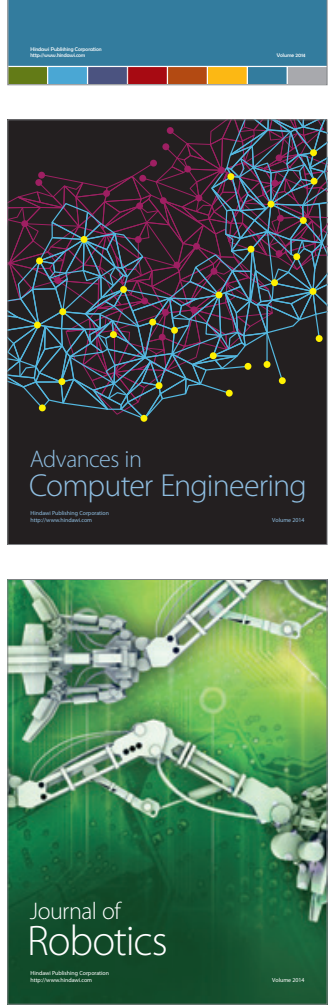
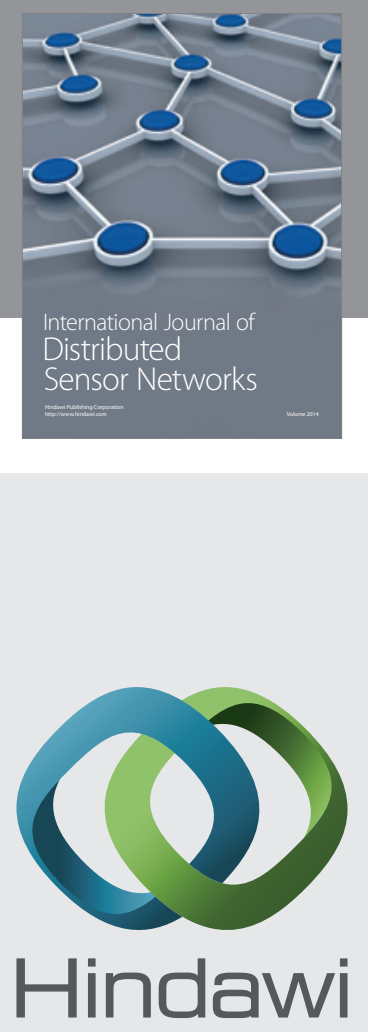

Submit your manuscripts at

http://www.hindawi.com

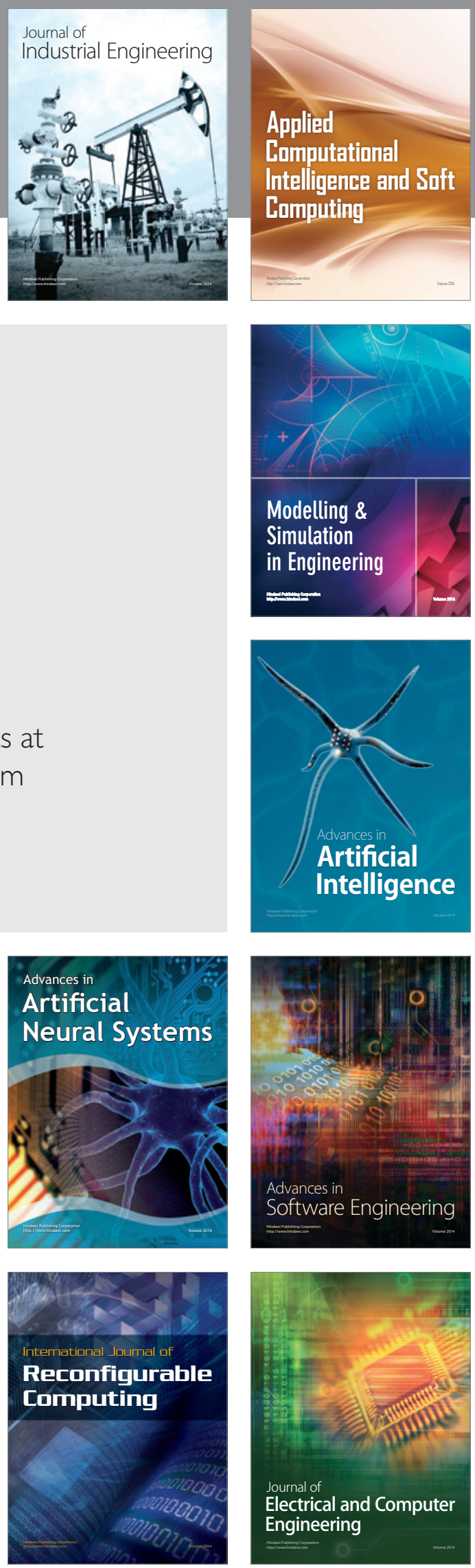